\title{
Quantum pumping in graphene with a perpendicular magnetic field
}

\author{
Rakesh P. Tiwari ${ }^{\mathrm{a})}$ and M. Blaauboer \\ Kavli Institute of Nanoscience, Delft University of Technology, Lorentzweg 1, 2628 CJ Delft, \\ The Netherlands
}

(Received 10 September 2010; accepted 29 November 2010; published online 17 December 2010)

\begin{abstract}
We consider quantum pumping of Dirac fermions in a monolayer of graphene in the presence of a perpendicular magnetic field in the central pumping region. The two external pump parameters are electrical voltages applied to the graphene sheet on either side of the pumping region. We analyze this pump within scattering matrix formalism and calculate both pumped charge and spin currents. The predicted charge currents are of the order of $1000 \mathrm{nA}$, which is readily observable using current technology. () 2010 American Institute of Physics. [doi:10.1063/1.3528457]
\end{abstract}

By periodically changing the confining potential of a quantum system, quantum pumping allows a net dc of carriers to flow in the absence of an applied bias. ${ }^{1-3}$ The possibility of generating electrical currents in solid-state nanostructures using this phenomenon has attracted considerable attention. ${ }^{4}$ The same phenomenon allows us to adiabatically generate spin-polarized currents in solid-state nanostructures, ${ }^{5}$ which is of great interest in the field of spintronics. Usually the pumped currents are small $(\sim 100 \mathrm{pA})$ and difficult to identify; as such, a small signal may be obscured by rectification. Indeed, quantum pumps realized so far are commonly believed to have demonstrated rectified currents. ${ }^{6-8}$ In this article we propose a quantum pump realized in graphene in the quantum Hall regime. We predict large pumped currents $(\sim 1000 \mathrm{nA})$ at modest values of gate voltages and magnetic fields, which increase the hope for unambiguous demonstration of quantum pumping.

Ever since the synthesis of high-quality graphene, ${ }^{9}$ there has been tremendous interest in the properties of this singlelayer form of carbon. ${ }^{10}$ Partly as a result of its electronic structure, ${ }^{9,11-13}$ graphene exhibits many unusual electronic and magnetic transport properties, such as a unique type of quantum Hall effect, ${ }^{9,14}$ ballistic conduction by massless Dirac fermions, ${ }^{9,14}$ a size-dependent band gap, ${ }^{15}$ and large magnetoresistance. ${ }^{13,16-18}$ Recently some quantum pumps based on graphene were proposed. Zhu and Chen ${ }^{19}$ considered a monolayer charge pump with two gate voltages as pumping parameters. Prada et al. ${ }^{20}$ considered a similar charge pump and found that the current is pumped through evanescent modes in the pumping region. In our proposed pump, both charge and spin currents are pumped through traveling modes and the magnitudes are much larger $(\sim 1000 \mathrm{nA})$, making it more desirable for applications.

Figure 1 shows a schematic of the device we consider. On the left and right ends of the graphene sheet two external voltages $V_{1}(t)=V_{1}+\delta V_{1} \sin (\omega t)$ and $V_{2}(t)=V_{2}+\delta V_{2} \sin (\omega t$ $+\phi)$ are applied. An external field $B_{0} \hat{z}$ (perpendicular to the graphene sheet) is applied in the central part of the sheet of length $L$, which forms the pumping region. We consider short and wide pumps $[\operatorname{width}(W) \gg \operatorname{length}(L)]$ for which the microscopic details of the edges become insignificant and the pumped current is obtained by integrating over the transverse modes. Also, we assume that the dimensions of the pump are

${ }^{a)}$ Electronic mail: rapat82@gmail.com. much larger than the carbon-carbon lattice constant. In that case the intervalley tunneling is suppressed and it is sufficient to consider just one valley. ${ }^{21}$

We use the scattering matrix formalism for calculating the pumped current. ${ }^{3}$ We divide the system into three regions. In region $1(x<-L / 2)$ and region $3(x>L / 2)$ gate voltages $V_{1}(t)$ and $V_{2}(t)$ are applied, respectively. In these regions both up-spin and down-spin carriers obey the Dirac equation of motion described by the Hamiltonian $\mathcal{H}$ $=v_{F} \sigma \cdot \mathbf{p}+e V_{j}$, where $j=1$ for region 1 and $j=2$ for region 3 , $\mathbf{p}$ is the momentum operator, $v_{F}$ is the Fermi velocity, and $\sigma=\left(\sigma_{x}, \sigma_{y}, \sigma_{z}\right)$ represents the three Pauli matrices. In region 2 , we describe the effect of the magnetic field $B_{0}$ and include the effect of Zeeman splitting. We choose the Landau gauge, such that the vector potential is $\mathbf{A}(x)=\left(0, B_{0} x, 0\right)$. Then in region 2 the Hamiltonian for up-spin and down-spin carriers is $\mathcal{H}_{\uparrow}=v_{F} \sigma \cdot[\mathbf{p}+e \mathbf{A}(x)]+g^{*} \mu_{B} B_{0} / 2 \quad$ and $\quad \mathcal{H}_{\downarrow}=v_{F} \sigma \cdot[\mathbf{p}$ $+e \mathbf{A}(x)]-g^{*} \mu_{B} B_{0} / 2$, respectively. Here $g^{*}$ is the spin $g$-factor (assumed to be 2) (Ref. 22) of the carriers and $\mu_{B}$ represents the Bohr magneton. To simplify the notation we introduce dimensionless units: $l_{B}=\sqrt{\hbar /\left(e B_{0}\right)}, \quad \mathbf{r} \rightarrow l_{B} \mathbf{r}, q$ $\rightarrow q / l_{B}, k_{x} \rightarrow k_{x} / l_{B}, E \rightarrow E_{0} E$, and $E_{0}=\hbar v_{F} / l_{B} . \hbar k_{x}$ represents the momentum along the $x$-axis.

In the adiabatic regime and for (bi)linear response the spin-dependent pumped current through each mode into the left lead is given by ${ }^{3}$

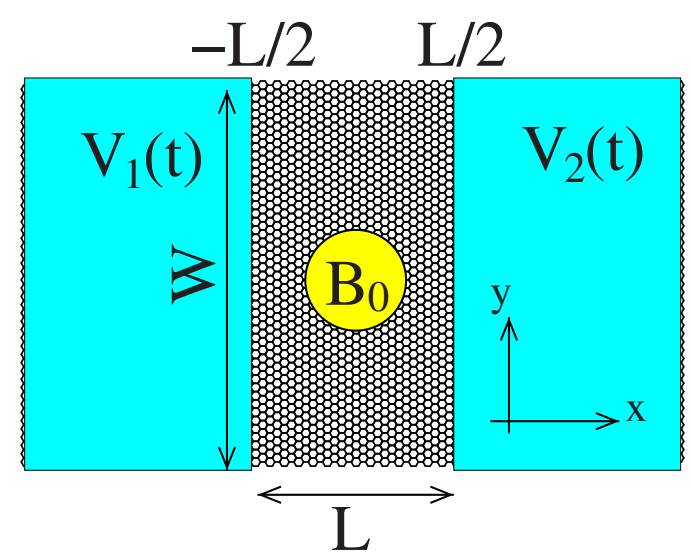

FIG. 1. (Color online) Schematic of the proposed quantum pump. An external field $B_{0} \hat{z}$ is applied in the central pumping region. The gate voltages $V_{1}(t)$ and $V_{2}(t)$ are the two pumping parameters. 


$$
I_{p}^{\sigma}=\frac{\omega e \sin \phi \delta V_{1} \delta V_{2}}{2 \pi} \operatorname{Im}\left[\frac{\partial r^{\sigma *}}{\partial V_{1}} \frac{\partial r^{\sigma}}{\partial V_{2}}+\frac{\partial t^{\sigma^{*}}}{\partial V_{1}} \frac{\partial t^{\sigma}}{\partial V_{2}}\right] .
$$

Here $r^{\sigma}$ and $t^{\sigma}, \sigma=\uparrow$ or $\downarrow$, represent the coefficients for reflection and transmission of carriers with spin $\sigma$ into the left reservoir (a similar expression can be derived for pumping into the right reservoir). The pumped charge current is $I_{p}^{\text {charge }}=I_{p}^{\uparrow}+I_{p}^{\downarrow}$ and spin current is $I_{p}^{\text {spin }}=I_{p}^{\uparrow}-I_{p}^{\downarrow}$. To calculate $r^{\uparrow}$ and $t^{\uparrow}$, we write down the wave functions in the three regions corresponding to the respective Hamiltonians, and then demand that the wave function is continuous across the boundaries at $x=-L / 2$ and $x=L / 2$. Due to the translational invariance of the geometry in the transverse direction, the momentum along the $y$-axis is conserved and is denoted as $\hbar q$. In region 1 the wave function is given by

$$
\Psi_{1}=e^{i q y}\left(\begin{array}{c}
e^{i k_{x}(x+L / 2)}+r^{\uparrow} e^{-i k_{x}(x+L / 2)} \\
e^{i k_{x}(x+L / 2)} e^{i \phi_{1}}+r^{\uparrow} e^{-i k_{x}(x+L / 2)} e^{-i \phi_{1}}
\end{array}\right),
$$

where $\tan \phi_{1} \equiv q / k_{x}$ and the energy of these excitations is given by $E=\left(e V_{1}+\hbar v_{F} \sqrt{k_{x}^{2}+q^{2}}\right) / E_{0}$. Similarly the wave function in region 3 is given by

$$
\Psi_{3}=t^{\uparrow} e^{i q y}\left(\begin{array}{c}
e^{i k_{x}^{\prime}(x-L / 2)} \\
e^{i k_{x}^{\prime}(x-L / 2)} e^{i \phi_{2}}
\end{array}\right),
$$

where $\tan \phi_{2} \equiv q / k_{x}^{\prime}$ and the energy of these excitations is given by $E=\left(e V_{2}+\hbar v_{F} \sqrt{k_{x}^{\prime 2}+q^{2}}\right) / E_{0}$. In the central region the wave function can be written as a linear combination of Weber functions, ${ }^{23}$

$$
\Psi_{2}=e^{i q y}\left(\begin{array}{c}
C_{1} D_{p-1}(z)+C_{2} D_{p-1}(-z) \\
\frac{i \sqrt{2}}{E}\left[C_{1} D_{p}(z)-C_{2} D_{p}(-z)\right]
\end{array}\right),
$$

where $\quad z=\sqrt{2}(x+q) \quad$ and $\quad p=\left[E-\left(g^{*} \mu_{B} \underline{B}_{0} / 2 E_{0}\right)\right]^{2} / 2$. Setting $\quad \alpha_{3}=D_{p-1}[\sqrt{2}(q-L / 2)], \quad \alpha_{4}=D_{p-1}[\sqrt{2}(-q+L / 2)]$, $\gamma_{1}=\sqrt{2} / E D_{p}[\sqrt{2}(q-L / 2)], \quad \gamma_{2}=\sqrt{2} / E D_{p}[\sqrt{2}(-q+L / 2)]$, $\omega_{1}=D_{p-1}[\sqrt{2}(q+L / 2)], \quad \omega_{2}=D_{p-1}[\sqrt{2}(-q-L / 2)], \quad \eta_{1}$ $=\sqrt{2} / E D_{p}[\sqrt{2}(q+L / 2)]$, and $\eta_{2}=\sqrt{2} / E D_{p}[\sqrt{2}(-q-L / 2)]$, we find

$$
\begin{aligned}
t^{\uparrow} & =\frac{2 i\left(\eta_{2} \omega_{1}+\eta_{1} \omega_{2}\right) \cos \phi_{1}}{f^{+} g^{+}-f^{-} g^{-}}, \\
r^{\uparrow} & =\frac{f^{-} h^{+}-f^{+} h^{-}}{f^{+} g^{+}-f^{-} g^{-}},
\end{aligned}
$$

where $f^{+}=i \eta_{2}+\omega_{2} e^{i \phi_{2}}, \quad f^{-}=i \eta_{1}-\omega_{1} e^{i \phi_{2}}, \quad g^{+}=i \gamma_{1}+\alpha_{3} e^{-i \phi_{1}}$, $g^{-}=i \gamma_{2}-\alpha_{4} e^{-i \phi_{1}}, h^{+}=i \gamma_{2}+\alpha_{4} e^{i \phi_{1}}$, and $h^{-}=i \gamma_{1}-\alpha_{3} e^{i \phi_{1}}$. Similar expressions are found for $r^{\downarrow}$ and $t^{\downarrow}$, the only difference being that $p=\left[E+\left(g^{*} \mu_{B} B_{0} / 2 E_{0}\right)\right]^{2} / 2$ instead of $p=[E$ $\left.-\left(g^{*} \mu_{B} B_{0} / 2 E_{0}\right)\right]^{2} / 2$.

Now we present the numerical results for the pumped charge and spin currents obtained using the above expressions for the reflection and transmission coefficients. The current is calculated using Eq. (1) and we show the total pumped currents which are obtained by integrating over all the modes, i.e., $I_{p, T}^{\text {charge }}=\int_{-\pi / 2}^{\pi / 2} \cos \left(\phi_{1}\right) I_{p}^{\text {charge }} d \phi_{1}$ and $I_{p, T}^{\text {spin }}$ $=\int_{-\pi / 2}^{\pi / 2} \cos \left(\phi_{1}\right) I_{p}^{\mathrm{spin}} d \phi_{1}$. We choose $W=5 \mu \mathrm{m}, L=0.5 \mu \mathrm{m}$, $V_{1}=V_{2}=0.1 \mathrm{~V}, \quad \delta V_{1}=\delta V_{2}=0.1 \mathrm{mV}, \quad v_{F}=10^{6} \mathrm{~m} / \mathrm{s}$, and $\omega /(2 \pi)=5 \mathrm{GHz} \cdot{ }^{19,22}$ The phase difference between the two external voltages $\phi$ is chosen to be $\pi / 2$ so as to maximize
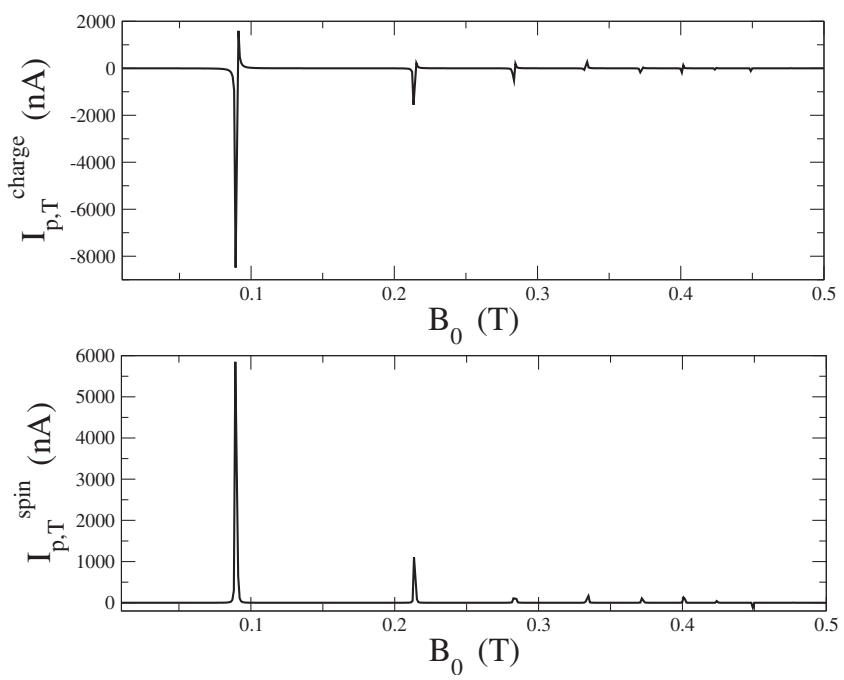

FIG. 2. Upper panel: pumped charge current as a function of $B_{0}$. Lower panel: pumped spin current as a function of $B_{0}$.

the pumped current. First we calculate the pumped current for fixed energy $E=\mu=100.1 \mathrm{meV}$. $\mu$ represents the Fermi energy in the two leads and the single particle state energy in the central pumping region. All these energies are taken to be equal in order to eliminate the possibility of generating current by applied external bias and secure energy-conserved tunneling. The temperature is set to zero in all our calculations and we ignore electron relaxation processes. $\mu$ is chosen to be $100.1 \mathrm{meV}$ in order to include the contribution of the lowest lying excitations for $V_{1}=V_{2}=0.1 \mathrm{~V}$ and $\delta V_{1}$ $=\delta V_{2}=0.1 \mathrm{mV}$.

The upper panel of Fig. 2 shows the pumped charge current as we change the magnetic field for the above mentioned parameters. The magnetic field is varied from 0.01 to $0.5 \mathrm{~T}$. As expected, the pumped currents are very sensitive to the magnetic field. The pumped current is large when the magnetic length $l_{B}$ is comparable to the dimensions of the pump. For $B_{0}=0.1 \mathrm{~T}, l_{B} \sim 100 \mathrm{~nm}$ and we see large values of the pumped currents $(\sim 1000 \mathrm{nA})$. As we increase the magnetic field, the magnetic length decreases and also the total pumped current decreases. The lower panel of Fig. 2 shows the calculated spin current for the same parameters. In general the pumped spin current is smaller than the pumped charge current. We believe that the enhancement of the pumped current in the proposed pump, compared to previously studied pumps without magnetic field, ${ }^{19,20}$ is due to interference arising from the magnetic length and the dimensions of the pump.

To compare the calculated pumped currents with rectified currents we calculate the conductance as a function of magnetic field for the same parameters (see Fig. 3). The conductance for up-spin carriers is given by $G$ $=G_{0} \int_{-\pi / 2}^{\pi / 2} \cos \left(\phi_{1}\right) t^{\dagger *} t^{\dagger} d \phi_{1}$ with $G_{0}=2 e^{2} k_{F} W /(\pi h)$, where $k_{F}$ is the Fermi wave-vector. The conductance for down-spin carriers (not shown) is almost identical. Peaks in the conductance correspond to a large jump in pumped currents, and the pumped charge current changes sign around that peak. This can be used to distinguish between any rectification current and the pumped current.

Finally we comment on the experimental realization of this pump. Fabricating metallic gates on graphene nanostructures with widths ranging from 10 to $100 \mathrm{~nm}$ and lengths of 


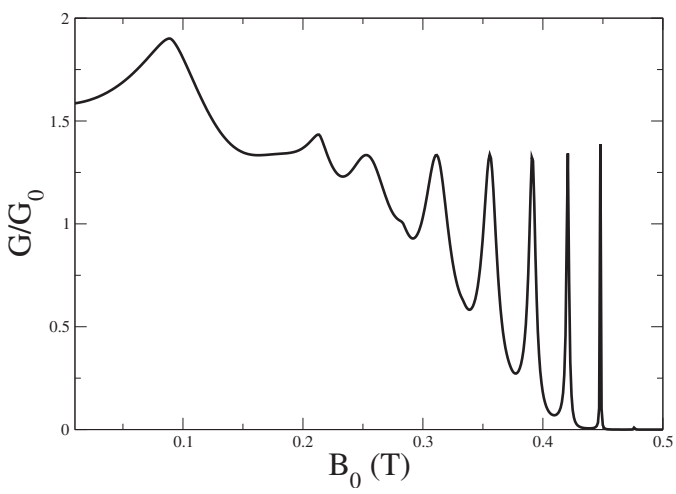

FIG. 3. Conductance $G / G_{0}$ as a function of $B_{0}$

1-2 $\mu \mathrm{m}$ can be achieved using current technologies. ${ }^{11,15}$ Perhaps the most difficult part will be to apply local magnetic fields of strengths up to $0.5 \mathrm{~T}$ in the central pumping region although efforts have already been made in this direction, for example, using nanomagnets. ${ }^{24}$ The latter can in principle be embedded under the central pumping region of the graphene pump. It should be noted that Eq. (1) represents the current pumped throughout one whole pumping cycle with no external bias applied. In practice because of the phase difference $\phi$ between $V_{1}(t)$ and $V_{2}(t)$ (chosen to be $\pi / 2$ ), there will be a small bias across the pump. The pump cycle can be chosen in a symmetric way such that the bias reverses after half of the period and any rectification current over the whole cycle is canceled.

To summarize, we have proposed a quantum pump based on graphene in a perpendicular magnetic field. The pumped current is carried by traveling modes and has a large magnitude $(\sim 1000 \mathrm{nA})$. We find that the pumped current has a characteristic dependence on $B_{0}$. Experimental verification of the pump properties would provide a much needed demonstration of the phenomenon of quantum pumping.

This research was supported by the Dutch Science Foundation NWO/FOM.
${ }^{1}$ M. Büttiker, H. Thomas, and A. Prêtre, Z. Phys. B: Condens. Matter 94, 133 (1994).

${ }^{2}$ B. Spivak, F. Zhou, and M. T. Beal Monod, Phys. Rev. B 51, 13226 (1995).

${ }^{3}$ P. W. Brouwer, Phys. Rev. B 58, R10135 (1998).

${ }^{4}$ B. L. Altshuler and L. I. Glazman, Science 283, 1864 (1999).

${ }^{5}$ E. R. Mucciolo, C. Chamon, and C. M. Marcus, Phys. Rev. Lett. 89, 146802 (2002).

${ }^{6}$ M. Switkes, C. M. Marcus, K. Campman, and A. D. Gossard, Science 283, 1905 (1999).

${ }^{7}$ S. K. Watson, R. M. Potok, C. M. Marcus, and V. Umansky, Phys. Rev. Lett. 91, 258301 (2003).

${ }^{8}$ P. W. Brouwer, Phys. Rev. B 63, 121303(R) (2001).

${ }^{9}$ K. S. Novoselov, A. K. Geim, S. V. Morozov, D. Jiang, M. I. Katsnelson, I. V. Grigorieva, S. V. Dubonos, and A. A. Firsov, Nature (London) 438, 197 (2005).

${ }^{10}$ A. H. Castro Neto, F. Guinea, N. M. R. Peres, K. S. Novoselov, and A. K. Geim, Rev. Mod. Phys. 81, 109 (2009).

${ }^{11}$ Y. Zhang, J.-W. Tan, H. L. Stormer, and P. Kim, Nature (London) 438, 201 (2005).

${ }^{12}$ C. Berger, Z. Song, X. Li, X. Wu, N. Brown, C. Naud, D. Mayou, T. Li, J. Hass, A. N. Marchenkov, E. H. Conrad, P. N. First, and W. A. de Heer, Science 312, 1191 (2006).

${ }^{13}$ A. K. Geim and K. S. Novoselov, Nature Mater. 6, 183 (2007).

${ }^{14}$ M. S. Purewal, Y. Zhang, and P. Kim, Phys. Status Solidi B 243, 3418 (2006).

${ }^{15}$ M. Y. Han, B. Ozyilmaz, Y. Zhang, and P. Kim, Phys. Rev. Lett. 98, 206805 (2007).

${ }^{16}$ E. W. Hill, A. K. Geim, K. Novoselov, F. Schedin, and P. Blake, IEEE Trans. Magn. 42, 2694 (2006).

${ }^{17}$ S. Cho and M. S. Fuhrer, Phys. Rev. B 77, 081402 (2008).

${ }^{18}$ R. P. Tiwari and D. Stroud, Phys. Rev. B 79, 165408 (2009).

${ }^{19}$ R. Zhu and H. Chen, Appl. Phys. Lett. 95, 122111 (2009).

${ }^{20}$ E. Prada, P. San-Jose, and H. Schomerus, Phys. Rev. B 80, 245414 (2009).

${ }^{21}$ T. Ando and T. Nakanishi, J. Phys. Soc. Jpn. 67, 1704 (1998).

${ }^{22}$ Y. Zhang, Z. Jiang, J. P. Small, M. S. Purewal, Y.-W. Tan, M. Fazlollahi, J. D. Chudow, J. A. Jaszczak, H. L. Stormer, and P. Kim, Phys. Rev. Lett. 96, 136806 (2006).

${ }^{23}$ M. Ramezani Masir, P. Vasilopoulos, A. Matulis, and F. M. Peeters, Phys. Rev. B 77, 235443 (2008)

${ }^{24}$ V. Kubrak, A. Neumann, B. L. Gallagher, P. C. Main, M. Henini, C. H. Marrows, and B. J. Hickey, J. Appl. Phys. 87, 5986 (2000); K. S. Novoselov, A. K. Geim, S. V. Dubonos, Y. G. Cornelissens, F. M. Peeters, and J. C. Maan, Physica E (Amsterdam) 12, 244 (2002); M. PioroLadrière, T. Obata, Y. Tokura, Y.-S. Shin, T. Kubo, K. Yoshida, T. Taniyama, and S. Tarucha, Nat. Phys. 4, 776 (2008). 